\title{
Smart wall by wireless sensor network toward building energy optimization
}

\author{
Asmaa Doublali ${ }^{1, *}$, Abdlilah Jilbab ${ }^{1}$, Chakib Bojji ${ }^{1}$, and Rachida Idchabani $^{2}$ \\ ${ }^{1}$ Electronics Sensors,Systems and Nanobiotechnologies ,ENSAM Rabat, Mohammed V university in Rabat, Morocco \\ ${ }^{2}$ Dep of process engineering, Superior National School of Mines, Mohammed V university in Rabat, Morocco
}

\begin{abstract}
Buildings design and operation are responsible for occupant comfort. Buildings facades and walls can be engineered to control solar energy for photovoltaic electricity generation, daylighting, heating, ventilation, thermal insulation, and energy storage. Adaptive facades and intelligent walls integrate realtime control technologies to adapt to the occupant's requirements and preferences. Data transmission and information control in the modern building are gaining increasing importance. Implementing wireless network systems based on wireless communication technologies and protocols with low energy demand is crucial. This paper presents the simulation of wireless networks based on Bluetooth Low Energy (BLE) transmission nodes with an energy harvesting system solution. The results showed the performance of the wireless sensor network model toward the solar energy harvesting solution.
\end{abstract}

Keywords - Bluetooth Low Energy, Wireless Sensor Network, Energy Harvesting, modern building, Bit Error Rate.

\section{Introduction}

Building Energy utilization represents $30 \%$ to $40 \%$ of the worldwide energy interest. With the growing population, limiting energy utilization has become essential for environmental issues. Even though the late research shows that "modern-day building" helped reduce energy consumption and carbon emissions [1], [2], a huge discrepancy was denoted in building's electricity demands and energy consumption [3]. For that reason, today's buildings are equipped with accurate and efficient sensing and communication technologies which play an important role in the storage and release of energy, limiting the overall energy utilization and ensuring the general comfort and safety of the habitants [4]. Technologies used for environmental monitoring in buildings could be described in a large group of sensor technologies with different functionalities and levels of complexities, such as temperature, humidity, light, carbon dioxide, occupancy, and airflow sensors. In addition, various communication technologies, such as Ethernet, Modbus, BACnet, Power Line Carrier, WIFI, Bluetooth, EnOcean, 6LoWPAN, LoRaWAN and Z-Wave are essential to complement these sensors. Communication technologies and protocols play a significant role in the integration of smart homes and buildings. These communication technologies guarantee secure and reliable communication, monitoring and storage of sensor information, data processing and communication in buildings management systems, and the functioning of the white goods. Over the last years the wired technologies, such as Ethernet, serial communication, and local area network were the most useful ones because they tend to be more secure, have fast data transfer speeds, and tend to be more reliable. Although recent literature [4], [5] suggests that wireless technologies, such as WIFI, Zigbee, Bluetooth, BLE, Etc.. are widely used due to the advances made in speed and security. Even though, both of the stated technologies have its utility and place in the market, it is essential to identify your respective requirement before making the choice.

Smart homes and buildings consist of data collection, data storage, data analysis, and visualization components. Automation actions in the building, like fixing the indoor environmental conditions (temperature, humidity) according to the user preference, turning lights on and off without human intervention, tracking home consumable products, improving the overall comfort, and making life easier. All these processes require collecting data from a distributed array of sensors. Wireless sensor networks (WSNs) give a potential answer for this issue[6]. WSN consist of hundreds to thousands of wireless nodes distributed within a geographical area. The wireless nodes collect information and supply it to the central node for further processing[7]. This large number of nodes formed one network. However, applications of

\footnotetext{
*Corresponding author: asmaa_doublali@um5.ac.ma
} 
WSN in building automation are expected to use a large number of nodes, even in simple applications like light and shutter switches with not many temperature sensors per room will cumulate in around a hundred rooms to thousands of nodes within the entire building[8]. Implementing these networks in traditional prototyping and adjusting is not the right path, rather than network simulation and modeling. In this study we developed a WSN with an energy harvesting solution using Matlab and studied the system's performance.

\section{Network wireless protocol choice}

The wireless technologies support remote data transfer, sensing, and control, to set various levels of intelligence in the smart building. The energy efficiency is what makes a wireless protocol ideal for use. Most of the existing sensing nodes are battery-powered [9]-[15]; thus a low power feature is a basic need for building energy consumption and economic savings. Therefore, the most critical and commercially available wireless technologies used in building and forming a network in a WSN are Zigbee, WIFI, and BLE. For Zigbee (IEEE802.15.4) operates in $2.4 \mathrm{GHz}$ and $900 \mathrm{MHz}$ bands, intended to provide low data transfer rate (250kbps), low cost, low power networking over a short distance (10-100m). so, the Zigbee finds its applications in home automation, smart meter, and sensor readings. WIFI (IEEE802.11) is the most adopted wireless technologies in buildings. WIFI operates in different unlicensed ISM frequency bands $(2.4 \mathrm{GHz}, 3.5 \mathrm{GHz}$ and $5 \mathrm{GHz})$, provides high data rates (11-1300 Mbps), a coverage range typically up to $(50-70 \mathrm{~m})$ including high power consumption and interference challenges (overlapping with Zigbee and Bluetooth frequency range $2.4 \mathrm{GHz}$ ), but the security is the major painful obstacle for this technology. By contrast, BLE comes out as a good low-power and secure wireless technology, with a large coverage range (up to $50 \mathrm{~m}$ ), and is easily included in billions of small devices and smartphones. These features make BLE technology suited for building automation systems applications [4], [16].

\subsection{Related works}

There are several works in which BLE presents the low energy consumption compared to other wireless technologies in the literature. Karan Nair[6] showed that BLE consumes less power during operation and connects faster than Zigbee or NRF. Matti Siekkinen[17] measured and modeled the energy consumption of BLE and compared it to that of 802.15.4. The findings revealed that BLE used very little energy and had a good energy per bit sent ratio. Artem Dementyev[18] analyzed the power consumption of the Zigbee, BLE, and ANT protocols in a cyclic sleep node scenario. The BLE achieved the lowest power consumption among them. Elke Mackensen and Matthias Lai[19] conducted a study about BLE-based wireless sensors systems. They concluded that BLE is a promising wireless technology for wireless sensor systems, mainly driven by an energy harvester. Moreover, it ended with some key features of a BLEbased wireless sensor system, such as little electricity usage, a high data throughput rate, straightforward implementation of BLE-based wireless sensors due to the good stack support of transceiver companies. Zengtao Feng[16] studied the ability to reduce the energy consumption of the wireless sensor with continuous data collection with a strategy of data packaging transmission for BLE. BLE has a huge potential in turning into a significant innovation for the Internet of Things (IoT), small devices, and smart homes, compared to IEEE 802.15.4, which seeks support in mobile devices. BLE has a lower energy consumption than Zigbee, so it is the best choice for home automation applications [20].

\section{Simulation model}

Simulation models in this study were built using MATLAB. Simulink presents the best environment for multidomain simulation (testing a variety of timevarying systems) and model-based design [21]. The simulation method's strength is in investigating the impact of multiple physical layer variables such as signal to noise ratio, channel noise and interference Etc.

\subsection{Bluetooth Low Energy signal}

Bluetooth Low Energy (BLE), Bluetooth 4, or Bluetooth Smart is a new technology created by the Bluetooth SIG (Special Interest Group) to overcome the power consumption limitations of the classic Bluetooth technology and for limited communication [22], [23]. The system diagram used to generate the Bluetooth Low Energy signal is illustrated in fig. 1. 

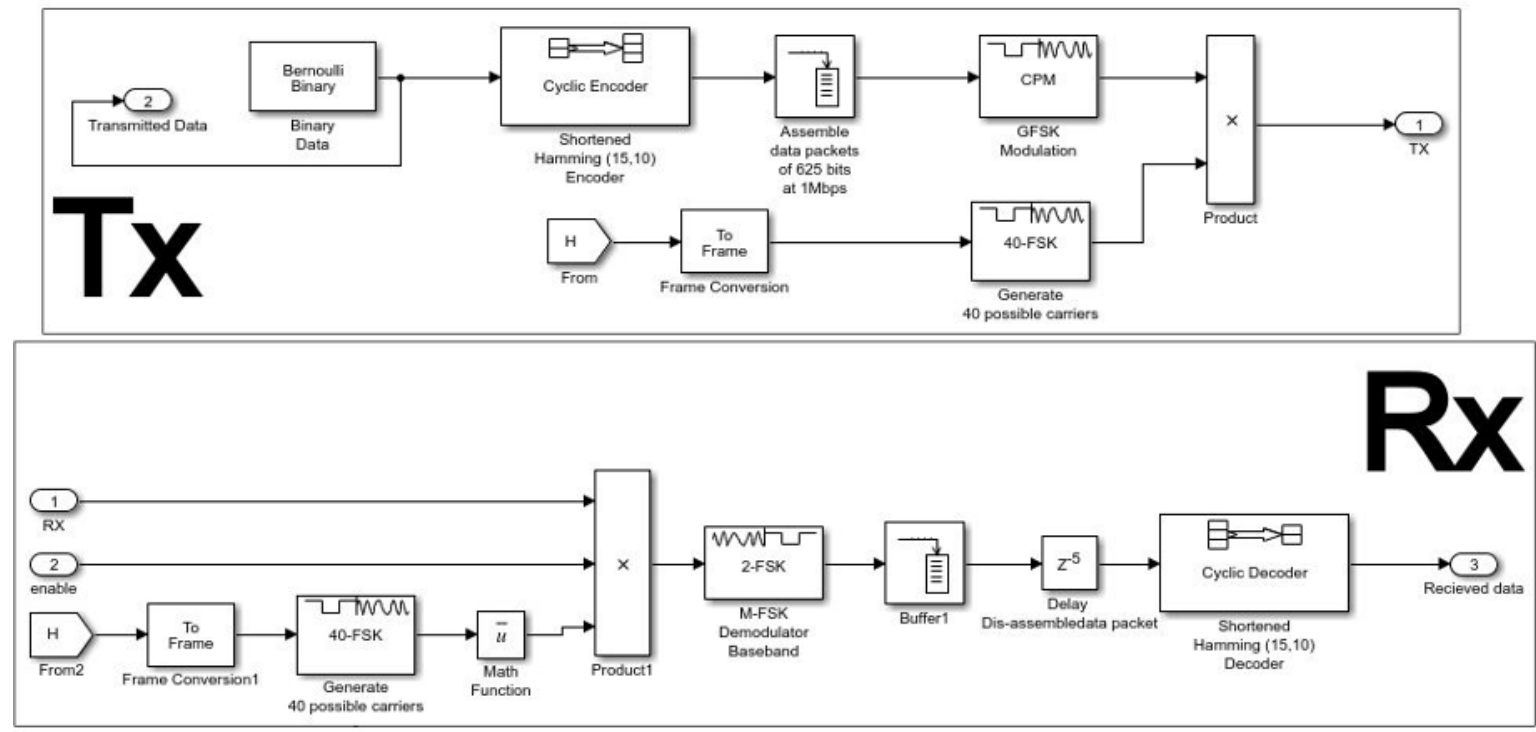

Fig. 1. Simulink model of BLE Physical layer.

The transmitter part is designed to collect 62500 bits at 1 Megabit per second $(\mathrm{Mb} / \mathrm{s})$. Those packets are then routed to Gaussian frequency shift keying (GFSK) modulator with a modulation index of 0.5 , there are 100 samples per symbol, and the pulse length is one bit per symbol. GFSK modulator is implemented with a frequency hopping mechanism. The purpose of frequency hopping over $40 \mathrm{MHz}$ frequency range (RF channels of BT LE $40(\mathrm{f}=2402+\mathrm{k} * 2 \mathrm{MHz}, \mathrm{k}=0, \ldots . ., 39))$ is to avoid interference with other devices transmitting in the band with a hop rate of $1600 \mathrm{~Hz}$. A random data channel between 0 and 36 is chosen to ensure that there are no overlapping channels [24]-[27].

\subsection{WSN model with optimization solution}

WSN model was built as shown in Fig. 2. The network consists of three sensor nodes with a solar energy harvesting solution communicating to each other using BLE technology which is considered the backbone of transmission operation. The three signals are sent from the sensor nodes transmitter part to the node's receiver part, the information obtained by receivers are used to estimate the BLE performance. Noise and interference are added to the signals to simulate the AWGN channel effect and measure Bit Error Rate (BER).

Here is a description of the proposed model:

The nodes switch section is implemented to decide between the three sensor nodes, which one to enable. The sensor nodes section comprises three subsystems named node1, node2 and node3; each node presented the transmitter and receiver sections of the block diagram illustrated in Fig.1. The transmitter TX converts the baseband information using the frequency hopping to the frequency modulated carrier. Contrary, the receiver RX decodes and demodulates using the same frequency hopping. The frequency hopping code is generated using the randi function.

The block bus creator/selector is used to create the output signals from the three nodes, TX1, TX2 and TX3 and select the transmitted one; in this case, TX1 is the transmitted signal. TX1 signal is transmitted over additive white gaussian noise channel (AWGN). The channel output is the input signal RX for the three nodes. $\mathrm{RX} 1, \mathrm{RX} 2$ and $\mathrm{RX} 3$ are the received data from the nodes.

In the BER calculation section, the received data RX1, RX2 and RX3 are compared to the transmitted data generated using the Bernoulli Binary block to analyze and check the accuracy of the transmitter and receiver communication.

The solar energy harvesting subsystem is implemented in the first sensor node. The output of the energy subsystem is transferred to the input sensor node using the rate transition block.

The solar energy harvesting system (SEHS) model as shown in Fig.3, is based on the overall system description presented in[28], [29]. Pulse Width Modulation (PWM) and maximum power point tracking (MPPT) control techniques have been analyzed and compared. From the comparison of the MATLAB simulation results, the efficiency of the MPPT based solar energy harvester system is better than the PWM controlled system efficiency. In general, a SEHS consist of [28]:

\section{- Solar cell efficiency}

- DC-DC converter

- MPPT design

In this study, the SEHS model as shown in fig.3, comprises a solar panel module, boost converter, MPPT algorithm (perturb and observe(P\&O)), PWM generator and rechargeable battery.

The inputs of the PV panel module are solar variable irradiance and constant temperature. The output generated by the PV module is the PV current; this electrical energy is delivered into a DC-DC boost converter, which boosts the output voltage. The rechargeable battery is charged using the Boost converter output voltage. The WSN node is powered by the rechargeable battery. Simulation parameters for SEHS are presented in Table 1. 
Table 1. Simulation parameters.

\begin{tabular}{|c|c|}
\hline Parameter & value \\
\hline Capacitors $(\mathrm{C})$ & $1000 \mu \mathrm{F}$ \\
\hline Inductance $(\mathrm{L})$ & $0.5 \mathrm{mH}$ \\
\hline DC-DC converter & Boost Converter \\
\hline Battery type & $\mathrm{NiCd}$ \\
\hline Resistance of inductor $\left(\mathrm{R}_{\mathrm{L}}\right)$ & $0.5 \Omega$ \\
\hline
\end{tabular}
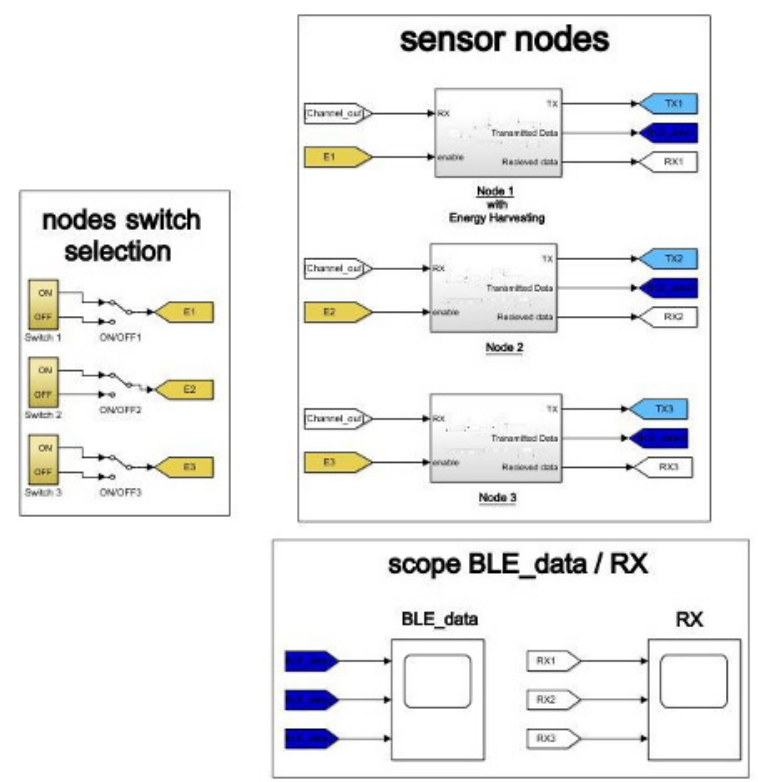
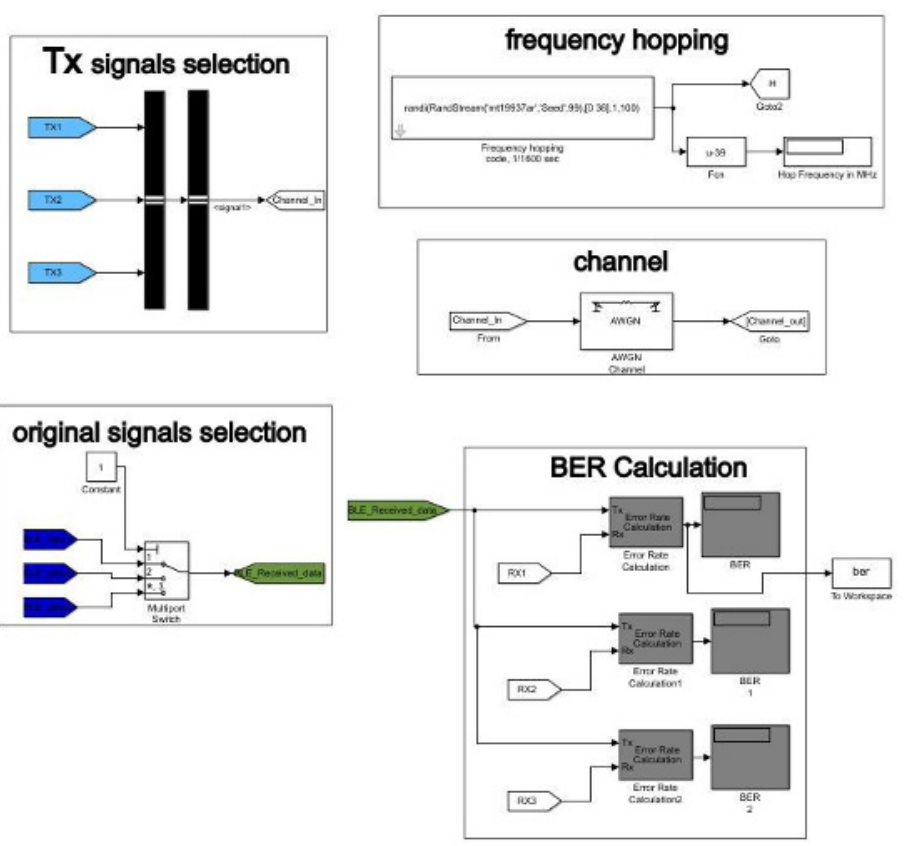

Fig. 2. WSN simulation model.

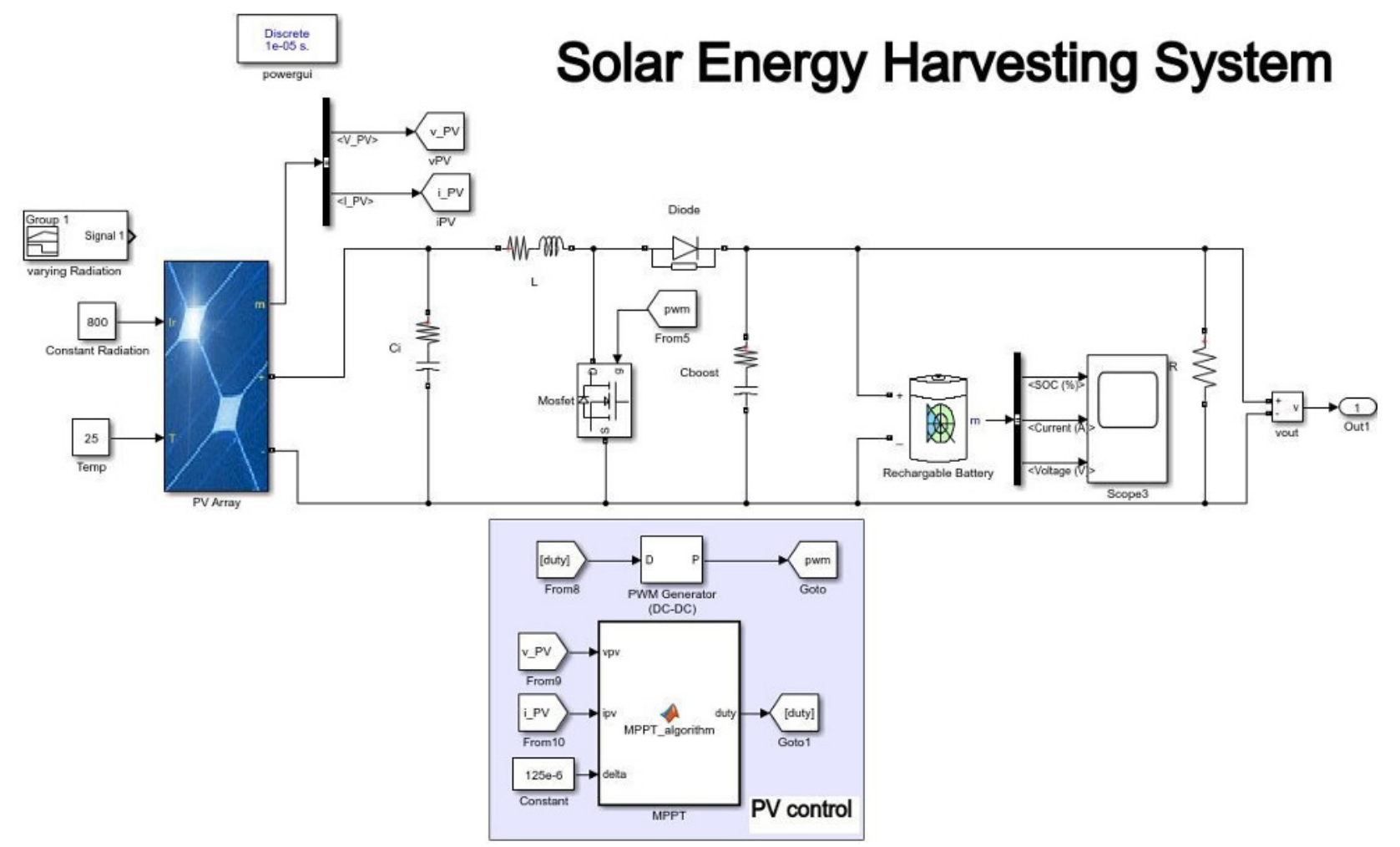

Fig. 3. SEHS simulation model.

\section{Simulation result}

The performance of the simulated system is evaluated using a bit error rate (BER) by Monte Carlo analysis, in 
which the system BER is plotted against the bit energy to noise ratio $(\mathrm{Eb} / \mathrm{No})$.

The performance of BLE and SEHS has been made only in AWGN channel to see how the BER changes with different values of solar radiations to the spectral noise density ratio (Eb/No). In Fig.4., the BER comparison between different solar radiations values in AWGN is given.

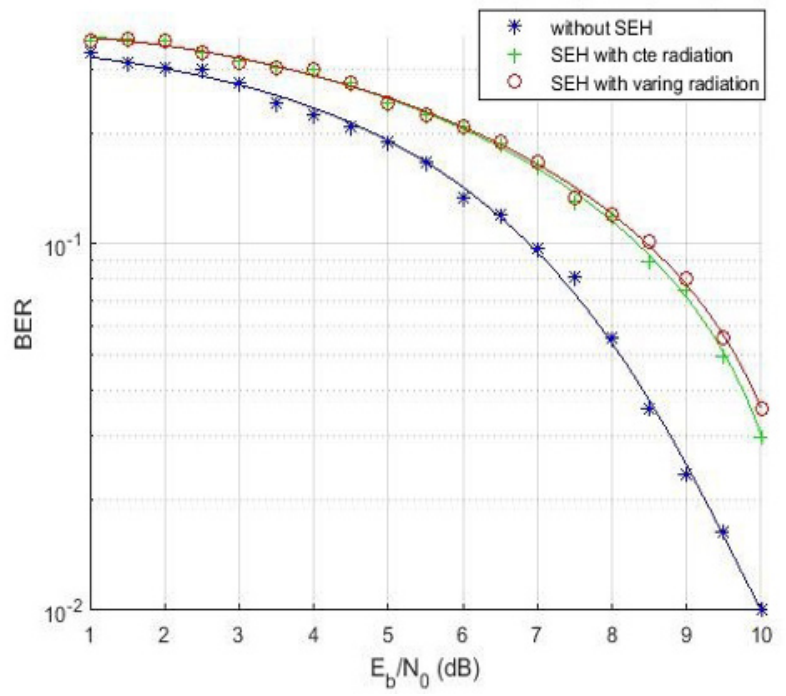

Fig. 4. BER performance in AWGN under variable solar irradiances

If we consider the case of a building for which the noise power is equal to the fifth of the BLE power signal $(\mathrm{Eb} / \mathrm{No}=5$, which corresponds to $7 \mathrm{~dB}$ point on the $\mathrm{X}$ axis), we consider a lower BER in the case where the WSN is without SEHS. To achieve the same BER, the case where WSN with SEHS would have to be $1,5 \mathrm{~dB}$ higher than the case without SEHS. The system is performing better in the case without SEHS.

\section{Conclusion}

Optimal energy use is the key factor for sustainable building operations. Therefore, the best combinations of building envelope materials (adaptive facades, intelligent walls), energy saving technologies, and optimization based on different climate conditions can significantly reduce energy consumption. In this context, we proposed sensor nodes in order to measure indoor/outdoor building climatic effects. The nodes are autonomous and powered by ambient solar photovoltaic energy to solve the limited battery energy problem.

We have simulated three nodes, which correspond to the external nodes. The transmission operations consist of Bluetooth Low Energy (BLE) technology, and for long network lifetime, an efficient solar energy harvesting system (SEHS) was added.

The simulation results come in the form of BER against SNR curves. It should be noted that the system performance degrades by changing the radiations of the SEHS. The communications are affected by different inputs of solar radiation. Further work will include adding more sensor nodes to the system to reveal all possible networking effects. Advanced algorithms could be implemented to study how stable and efficient the proposed approach is in the BLE communication and data transmission.

The research described in the paper was financially supported by Institut de Recherche en Energie Solaire Et Energies Nouvelles (IRESEN).

\section{References}

[1] M. H. Chung et E. K. Rhee, «Potential opportunities for energy conservation in existing buildings on university campus: A field survey in Korea », Energy and Buildings, vol. 78, p. 176182.

[2] AECOM, Aviva investors, et Balfour Beatty, «Carbon Reductions in Existing Non-Domestic Buildings », UK Green Building Council, Match (2011).

[3] Menezes, B. Richard, et B. Dino, «Analysis of electricity consumption for lighting and small power in office buildings ", in CIBSE Technical Symposium (2011), p. 15.

[4] H. Hayat et al., « The State-of-the-Art of Sensors and Environmental Monitoring Technologies in Buildings ", Sensors, vol. 19, no 17, p. 3648, août (2019).

[5] T. Grimard et L. Kieran, «Wireless Options Becoming More Prevalent with BAS », Facilitiesnet, janv. 29, (2016).

[6] K. Nair et al., " Optimizing power consumption in iot based wireless sensor networks using Bluetooth Low Energy », in 2015 International Conference on Green Computing and Internet of Things (ICGCIoT), Greater Noida, Delhi, India, oct. 2015, p. 589-593, Consulté le: juin 08, (2020).

[7] A. Jangra, «Wireless Sensor Network (WSN): Architectural Design issues and Challenges », vol. 02, no 09, p. 6, (2010).

[8] M. Galetzka, J. Haufe, M. Lindig, U. Eichler, et P. Schneider, "Challenges of simulating robust wireless sensor network applications in building automation environments $»$, in 2010 IEEE 15th Conference on Emerging Technologies \& Factory Automation (ETFA 2010), Bilbao, sept. (2010), p. 1-8.

[9] A. Mainwaring, J. Polastre, R. Szewczyk, D. Culler, et J. Anderson, «Wireless Sensor Networks for Habitat Monitoring », vol. pp. 88-97, p. 10 , sept. 2002.

[10] P. Zhang, C. M. Sadler, S. A. Lyon, et M. Martonosi, "Hardware design experiences in ZebraNet ", in Proceedings of the 2nd international conference on Embedded networked sensor systems - SenSys '04, Baltimore, MD, USA, (2004), p. 227.

[11] P. Dutta et al., « Trio: Enabling Sustainable and Scalable Outdoor Wireless Sensor Network Deployments », 5th International Conference on Information Processing in Sensor Networks, vol. pp. 407-415, p. 9, 2006.

[12] J. Taneja, J. Jeong, et D. Culler, « Design, Modeling, and Capacity Planning for Micro-solar 
Power Sensor Networks », in 2008 International Conference on Information Processing in Sensor Networks (ipsn 2008), St. Louis, MO, USA, avr. (2008), p. 407-418.

[13] D. Musiani, K. Lin, et T. S. Rosing, "Active sensing platform for wireless structural health monitoring ", in Proceedings of the 6th international conference on Information processing in sensor networks, New York, NY, USA, avr. (2007), p. 390-399.

[14] C. Alippi, R. Camplani, C. Galperti, et M. Roveri, «A Robust, Adaptive, Solar-Powered WSN Framework for Aquatic Environmental Monitoring », IEEE Sensors J., vol. 11, no 1, p. 45 -55, janv. (2011).

[15] X. Yue et al., «Development of an Indoor Photovoltaic Energy Harvesting Module for Autonomous Sensors in Building Air Quality Applications », IEEE Internet of Things Journal, vol. 4, no 6, p. 2092-2103, déc.(2017).

[16] Zengtao Feng, Lingfei Mo, et Meng Li, « Analysis of low energy consumption wireless sensor with BLE », in 2015 IEEE SENSORS, Busan, nov. (2015), p. 1-4.

[17] M. Siekkinen, M. Hiienkari, J. K. Nurminen, et J. Nieminen, «How low energy is bluetooth low energy? Comparative measurements with ZigBee/802.15.4 », in 2012 IEEE Wireless Communications and Networking Conference Workshops (WCNCW), Paris, France, avr. (2012), p. 232-237.

[18] A. Dementyev, S. Hodges, S. Taylor, et J. Smith, "Power consumption analysis of Bluetooth Low Energy, ZigBee and ANT sensor nodes in a cyclic sleep scenario », in 2013 IEEE International Wireless Symposium (IWS), Beijing, China, avr. (2013), p. 1-4.

[19] E. Mackensen, M. Lai, et T. M. Wendt, «Bluetooth Low Energy (BLE) based wireless sensors », in 2012 IEEE Sensors, Taipei, Taiwan, oct. (2012), p. 1-4.

[20] M. Collotta et G. Pau, «A Novel Energy Management Approach for Smart Homes Using Bluetooth Low Energy », IEEE J. Select. Areas Commun., vol. 33, no 12, p. 2988-2996, déc. (2015).

[21] Q. I. Ali, A. Abdulmaowjod, et H. M. Mohammed, «Simulation \& performance study of wireless sensor network (WSN) using MATLAB », in 2010 1 st International Conference on Energy, Power and Control (EPC-IQ), (2010), p. 307-314.

[22] C. Gomez, J. Oller, et J. Paradells, « Overview and Evaluation of Bluetooth Low Energy: An Emerging Low-Power Wireless Technology », Sensors, vol. 12, no 9, p. 11734-11753, août (2012).

[23] J. Tosi, F. Taffoni, M. Santacatterina, R. Sannino, et D. Formica, "Performance Evaluation of Bluetooth Low Energy: A Systematic Review », Sensors, vol. 17, no 12, p. 2898, déc. (2017).
[24] « Introduction to Bluetooth Device Testing From Theory To Transmitter and Receiver Measurements », sept. (2016).

[25] A. D'Souza, H. Viittala, M. Hamalainen, et L. Mucchi, «Performance Comparison Between ETSI SmartBAN and Bluetooth », in 2018 12th International Symposium on Medical Information and Communication Technology (ISMICT), Sydney, NSW, mars (2018), p. 1-5.

[26] M. B. Roslee et T. W. Lee, «IMPLEMENTATION OF RFID AND BLUETOOTH TECHNOLOGY FOR DATA TAGGING AND TRANSMISSION TO POINT OF SALE », PIER B, vol. 40, p. 185-200, (2012).

[27] M. R. Badiger, D. M. Nagaraja, D. M. Z. Kurian, et I. Rasheed, "Analysis, Design and Testing of Frequency Hopping Spread Spectrum Transceiver Model Using MATLAB - Simulink », vol. 3, no 2, p. 7.

[28] H. Sharma, A. Haque, et Z. A. Jaffery, « Solar energy harvesting wireless sensor network nodes: A survey », Journal of Renewable and Sustainable Energy, vol. 10, no 2, p. 023704, mars (2018).

[29] H. Sharma, A. Haque, et Z. Jaffery, « Modeling and Optimisation of a Solar Energy Harvesting System for Wireless Sensor Network Nodes », JSAN, vol. 7, no 3, p. 40, sept. (2018). 